\title{
Students' Perception of Mobile Learning in a Blended Collaborative Learning Framework in Mathematics
}

\author{
Anyor, Joseph Wuave PhD and Abah, Joshua Abah \\ Department of Science Education \\ University of Agriculture Makurdi \\ Benue State, Nigeria.
}

\begin{abstract}
Mobile phones have become ubiquitous in today's modern society. More Nigerians are finding the use of mobile technologies a normal aspect of their daily lives. At the heart of the trend are students, who do not only own mobile gadgets for the basic necessity of communication, but also for social status, leisure and a wide range of applications. The extent of penetration and utilization of mobile phones in schools has led educationists to explore the possibilities of harnessing their positive impact on students' educational experience. A device that is everywhere, every time with the learner can be used to encourage the learner to learn on-the-go. Mobile Learning provides the opportunity to extend students' learning experience beyond the walls of the classroom, thereby offering the learner greater participation and control of the learning process. This study considers the efficacy of mobile learning when blended into a conventional instructional system. The results of the study indicate a paradigm shift in favour of efficient inclusion of emergent technologies in instructional processes, particularly at the tertiary education level.
\end{abstract}

Keywords: Mobile Phones, Mobile Learning, M-Learning Technologies, Collaborative Learning, Internet access.

Recommended Citation

Anyor, J. W. \& Abah, J. A. (2014). Students' perception of mobile learning in a blended collaborative learning framework in mathematics. Benue Journal of Mathematics and Mathematics Education, 1(3), 20-27 


\section{Introduction}

The penetration of computer technology in society left indelible imprints on every facet of human existence. It revolutionizes human thinking and opened up access to a whole lot of possibilities. One of the spheres of impact of technology-enabled services is the education sector. In a 2009 white paper released by Research in Motion Limited (makers of Blackberry Smartphones), the higher education marketplace was said to be full of challenges ranging from dropping budgets to diversity in student demographics, which made it important to attract, engage, and retain students by enriching academic experience (RIM, 2009).

Improved internet bandwidth coupled with lack of time leads to enormous potential and demand for instantaneous services (Kumari and Singh, 2009). This implies that the quality of a service is now based on its immediate accessibility and ability to adapt to emerging trends. One of the most progressive ways for learning management services to keep up with the pace in advancement of Information and Communications Technology (ICT) is through Mobile Learning (m-learning).

At a glance, Mobile Learning refers to the ability to "learn on-the-go" as occasioned by the increasing penetration of mobile phones, personal digital assistants (PDAs) and Smartphones (Muyinda, Lubega \& Lynch, 2010). A learner can decide to utilize a learning medium and content based on personal requirements, interests, and constraints. Since mobile devices are truly portable communication media, the availability of learning content through mobile medium will definitely expedite the learning process.

Mobile Learning using handheld devices draws on the theory and practice of pedagogies used in technology enhanced learning (Traxler, 2009). The term covers the personalized, connected, and interactive use of handheld computers in classrooms and in collaborative learning. Collaborative learning, or working in groups, is a great way to involve students who require social engagement during the learning process. Peers can mentor one another while building community and social skills (Seehorn, 2013).

Much of the current research on the effectiveness of mobile learning has established that it is effective and can compliment various models of instruction delivery (Muyinda et al, 2010; Wishart, 2009). So far, these forms of utilization has focused on short-term small- 
scale pilots and trials in the developed countries of Europe, North America, and the Pacific Rim (Traxler, 2009), but that is not the case in developing countries where growth in computer-aided learning (CAL) deployment is characterized by low incomes and the inability to afford expensive PCs and Internet access (Mockus, Dawson, Edel-Malizia, Shaffer, Sung An, \& Swaggerty, 2011).

This study focuses on students overall satisfaction with and perception of mobile learning when used in a collaborative learning framework. The results of this non-experimental quantitative study represent the rapidly changing dynamics among higher education students in Nigeria.

\section{Mobile Learning}

In trying to arrive at a definition of mobile learning, one has to sift through complex bases introduced in terms of technologies and hardware. A sound definition should not be so constraining, technocentric and tied to current technological instantiations (Traxler, 2009). Care has to be taken, considering the fact that the world of Information and Communications Technology (ICT) is very dynamic.

The term Mobile Learning (m-learning) refers to the use of mobile and handheld devices, such as Personal Digital Assistants (PDAs), mobile telephones, laptops and tablet PC technologies in teaching and learning. Mobile Learning has severally been referred to as "knowledge on the move" occasioned by the increased portability, affordability and ease of use. According to the Mobile Learning Network (MoLeNET, 2012), Mobile Learning is "the exploitation of ubiquitous handheld technologies, together with wireless and mobile phone networks, to facilitate, support, enhance and extend the reach of teaching and learning". Mobile Learning allows for contextualization of learning as well as the mobility of the learner (Belshaw, 2012).

Mobile devices are getting cheaper by the day, and are gradually becoming a necessity for an average social life. Ogunlesi and Busari (2012) in a report for the CNN observed the sharp rise on access to telecommunication gadgets from a peasant 100, 000 phone lines (mostly owned by state-owned NITEL) over a decade ago to "close to 100 million mobile phone lines, making Nigeria Africa's largest telecoms market”. The idea behind the adoption of mobile learning by educationists is the shear availability of mobile 
phones among students who use them for different purposes ranging from phone calls and text messaging to social networking. Co-opting mobile devices for the purpose of learning through the provision of learning content via mobile medium will definitely expedite the learning process. Thus, mobile learning is a transformative opportunity and does represent a significant means of augmenting formal training.

According to Brandon (2011), m-learning makes it possible to think beyond formal instruction (especially, classroom-based, instructor-mediated events) to integrate learning strategy with overall performance and technology strategy, and to complement formal learning with informal learning, social learning and performance support. In most cases, m-learning does not fit in as an overall strategy of instruction delivery, but when adopted to complement existing instructional strategy it gives the desired yield. As such, mobile access is not so much a replacement for course work but rather as an enhancement to the blended learning experience.

Kumari et al (2009) observed that m-learning makes learning truly personalized as learners have the option to choose learning content based on their interest. Apart from being learner-centric, m-learning increases productivity, content consistency and motivation. There is an indication that not only does Mobile Learning engage students, and speak to them in a way that fits their lifestyle, it also prepares them to enter the modern workforce (RIM, 2009). The idea is that students who gain experience with realworld learning tools will have an advantage in the workforce.

\section{M-Learning Technologies}

Effective m-learning is more than simply repackaging existing e-learning (Quesinberry, 2011). It involves carefully selecting m-learning objects (MLO) which take cognizance of mobile device limitations (Muyinda et al, 2010). Such limitations include low resolution, tiny screens and keyboard, low bandwidth and power. This implies that technologies that enhance m-learning must include appropriate mobile devices, mobile software and mobile operating system.

Mobile Devices: The m-learning ecosystem is made up a wide variety of devices connected via different kinds of networks (Mockus et al,2011). Common devices include 
camera phones, smartphones, Personal Digital Assistants (PDAs), netbooks, tablet PCs and portable media players.

Almost all devices suitable for m-learning has capacity to make phone calls, send and receive text messages (SMS), connect to the internet through GPRS or cloud (wireless) services, and play multimedia files such as pictures, videos and mp3 audio. New generation mobile devices such as smartphones, PDAs and tablet PCs have an endless range of capacity, considering the suites of applications available from App Stores.

Mobile Software: Brandon (2011) observed that mobile devices are not only convenient and always with us, but they are the focus of the convergence of capability. Each device offers a combination of applications to support documents formatting, audio, e-mail, multimedia message service (MMS), social interaction and the World Wide Web.

With the design of the Java 2 Micro Edition (J2ME) by Sun Microsystems, software capability on mobile devices became a thing of convenience. J2ME is an Application Programming Interface (API) to develop software for small and resource constrained devices (Kumari et al, 2009). J2ME provides a standard Java run time environment for mobile devices which hitherto has no platform for launching applications. Hence, smaller hand held devices can now power sophisticated software such as web browsers (Opera Mini, UC Web, QQ, etc), E-book readers, dictionaries, PDF readers, e-mail apps (yahoomail, gmail, etc.), and social networking apps (facebook, 2go, Mxit, WhatsApp etc).

Currently, a good mobile device in hand means the world in hand. This is more so, considering the functionality and productivity tools available on mobile devices. As a result, mobile learning utilizes this ease of access to information to transform moments which would be otherwise "wasted" into useful time enriched with didactic contents (Pieri \& Diamantini, 2009).

Mobile Operating Systems: Operating systems (OS) available on mobile devices include Symbian OS, Microsoft Windows Mobile OS, Palm OS, Blackberry OS, Android OS, iOS, and Mac OS X. As expected, applications are not interchangeable across operating systems, hence the crucial role played by $\mathrm{J} 2 \mathrm{ME}$ in acting as a broker across some of the operating systems to enhance portability of applications in .jar extension. 


\section{Blended Collaborative Learning Framework}

The term collaborative learning refers to an instruction method in which students at various performance levels work together in small groups towards a common goal (Gokhale, 1995). Collaborative learning is a team process where members support and rely on each other to achieve an agreed upon goal.

With the increase in globalization of business, advances in technology and changes in organizational structure, much emphasis is being shifted to teamwork within any given workforce. If education must prepare young ones to contribute meaningfully to the larger society, then children in our schools should be exposed to the working structure of he outside world where survival is by efficacy of team spirit. This exposure only comes through strategies that mimic, to a large extent, the exact type of teamwork available in complex organizational structure. This is where collaborative learning comes into play. Collaborative learning is a relationship among learners that requires positive interdependence (a sense of sink or swim together), individual accountability (each of us has to contribute and learn) and interpersonal skills (communication, trust, leadership, decision making, and conflict resolution). These qualities of collaborative learning are obtained in a more glaring form when several instructional strategies are deployed together.

Blended learning is essentially just a blending of different learning methods and techniques. It allows students to select the best activities to suit their own pace, learning style and level, as well as time and place (RIM, 2009). With the right technology, learners can have a socially supported, exploratory and constructive learning experience literally at their fingertips.

The recent trend in learning delivery is blended learning, which combines different modes of learning to make learning more effective and engaging (Kumari et al, 2009). Blended learning marries the strengths of different learning strategies and helps to best fit the learning context in an interactive learning environment. Mobile learning can be incorporated into other modes of learning to enhance and sustain interest as well as support additional training materials. M-learning can activate knowledge before 
classroom training through explanatory exercises or summary of the main concepts (Quesinberry, 2011).

\section{Objectives of the Study}

The objective of this study is to find out students' perception of mobile learning when used in a blended collaborative instructional framework. Specifically, the study seeks to

1. ascertain the category of mobile devices readily available among students for mobile learning;

2. find out the activities mobile devices used for among students; and

3. find out students' perceptions of mobile learning when used in a blended collaborative learning framework.

\section{Research Questions}

This non-experimental quantitative study attempted to explore student's perception of mobile learning when used in a blended collaborative instructional framework. The following questions where raised to guide the study.

1. What are the mobile devices readily available among students for mobile learning?

2. What activities are mobile devices used for among students?

3. What are students' perceptions of mobile learning when used in a blended collaborative learning framework? 


\section{Methodology}

\section{Site and Sample Demographics}

The site for the study was the University of Agriculture Makurdi, located in Benue State, Nigeria. The population consisted of undergraduate students enrolled in B.Sc(Ed) Integrated Science programme offered by the University. The sample consisted of 300 Level students enrolled in the course: Applied Mathematics for Sciences.

The sampling technique used was purposive sampling. The study was limited to students enrolled in the course because they were exposed to the details of mobile learning by the researchers who happened to be the course's lecturers for the First Semester 2012/2013 academic session. The students were required to submit their mobile phone numbers and email addresses for the communication of mobile learning objects. Course contents were delivered via bulk SMS to all students on an average of two separate SMS per week. A typical text message reads: "Why are bullets more dangerous than guns? Kinematics is the study of the motion of objects." This message was sent via bulk SMS (with the course code as title) a few days before introducing Kinematics as a topic in class. Background research works were submitted by students via a central e-mail account created for the study, and Teacher-Marked Assignments are posted to students e-mails. Similar communications were also used in coordinating group projects.

\section{Data Collection}

Data was collected via the Students Mobile Learning Perception Questionnaire (SMLPQ). The SMLPQ was an adapted form of the World Campus Mobile Learning Research Survey designed at the Pennsylvania State University's Campus (Mockus et al, 2011). The SMLPQ consisted of two sections, A and B. Section A aimed at eliciting responses about mobile devices, while section B was on students' perception. The SMLPQ was administered on the subjects at the completion of the course work.

\section{Method of Data Analysis}

The method of data analysis used in this research was descriptive analysis using charts and simple percentages. 


\section{Results}

The results of the study are presented according to the research questions.

\section{Research Question 1}

What are the mobile devices readily available among students for mobile learning?

The categories of mobile devices available among students were classified into three main categories based on capacity and functionality. These are Basic Phones (calls and text messages), Features Phone (calls, text messages, user applications, GPRS Internet access, camera, video and music), and Smartphones (calls, text messages, 3G Internet, Wi-Fi, Camera, VGA Camera, QWERTY keyboard, Microsoft Office, Mobile Email, Mobile pdf reader, etc). The results indicate that $10 \%$ of respondents have Basic Phones, $20 \%$ have Features Phones, while 70\% have Smartphones.

\section{Research Question 2}

What activities are mobile devices used for among students?

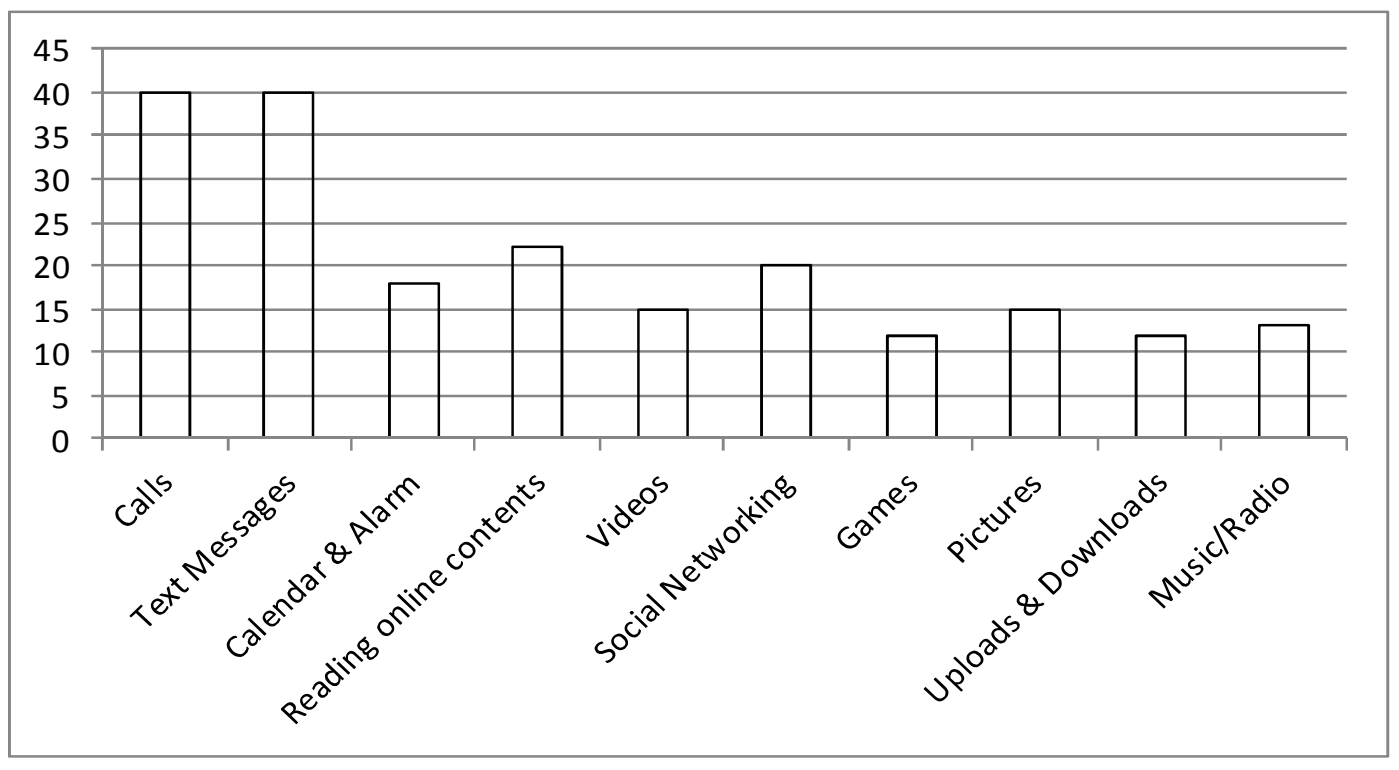

Figure 1: Activities Mobile Devices are used for.

Figure 1 shows the major activities students use their mobile devices for. The results also indicate that $5 \%$ of the students spends less than 1 hour daily using their mobile phones, $22.5 \%$ spends $1-2$ hours, $15 \%$ spends $2-3$ hours, and $57.5 \%$ spends 3 hours or more. On 
time spent online per day, $15 \%$ of respondents spend less than 15 minutes online daily, $37.5 \%$ spends up to 1 hour, $30 \%$ spends between 1 to 2 hours, and $17.5 \%$ spends more than 2 hours online daily. The distribution for students' mode of access to the internet is shown in Figure 2.

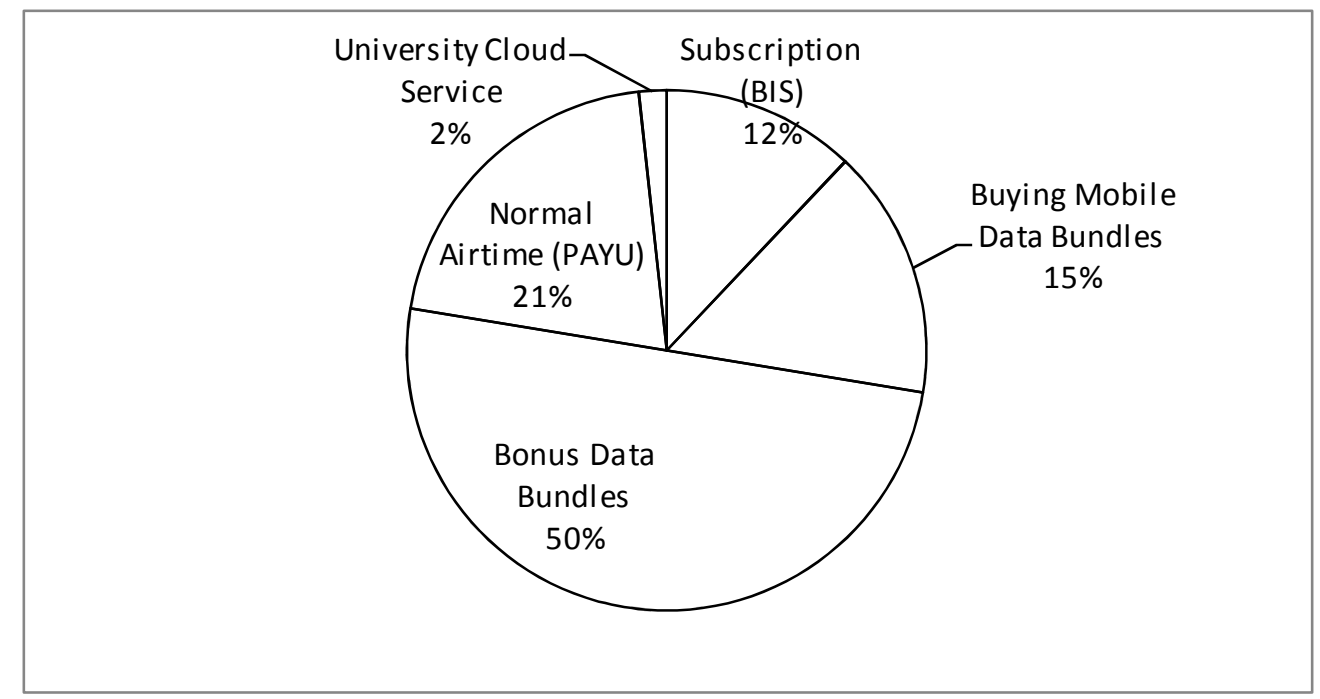

Figure 2: Students Mode of Access to the Internet

\section{Research Question 3}

What are students' perceptions of mobile learning when used in a blended collaborative learning framework?

Responses from the students indicate that $67.5 \%$ of students found the mobile learning approach very engaging, $25 \%$ found the approach somewhat engaging, and $7.5 \%$ found it not engaging. Also, 35\% of respondents prefer traditional instructional content, $20 \%$ prefers mobile instructional content, while $45 \%$ prefer a blend of both traditional and mobile instructional contents. $72.5 \%$ of students are more motivated in their study after learning experience in a blended collaborative framework, $20 \%$ felt about the same (indifferent), and $7.5 \%$ felt less motivated. 


\section{Discussions}

The results of the study as presented clearly affirm that mobile phone technology has become an indispensable part of students' daily life, with as much as $70 \%$ of students owning smartphones. A large proportion of students spend between above 3 hours using their mobile phones daily. This reality provides an ample opportunity to learn anytime and anywhere, as equally observed by Muyinda et al (2010). Activities pursued on mobile devices by students ranges from calls and SMS to reading of e-books and downloads of internet contents. There is also an indication that students are spending more time on the internet using mobile phones to access instructional contents than earlier thought.

A remarkable finding of this study is the multi-faceted modes of internet access available to students. The pie chart in Figure 2 shows that a greater percentage of students access the internet freely via bonus data bundles provided by mobile network providers like MTN, GLO, Etisalat, and Airtel as incentives for recharging airtime. The amount of free data depends on the SIM package and the amount of airtime recharged weekly. It is also obvious that students with the appropriate devices are beginning to benefit from the Universities Cloud (Wireless) Services.

The investigations in this research work revealed that students find the use of mobile learning in a blended collaborative learning framework to be very engaging and motivational. A larger proportion of the subjects of this study prefer a blend of both traditional instructional content and mobile learning contents.

\section{References}

Belshaw, D. (2012). What is Mobile Learning? Retrieved on 22 ${ }^{\text {nd }}$ March, 2013 from https://mobilelearninginfokit.pbworks.com/w/page/41751137/what\%20is\%20mob ile\%20learning

Brandon, B. (2011). Why You Need a Mobile Strategy. Retrieved on $22^{\text {nd }}$ March, 2013 from http://www.learningsolutionsmag.com/articles/682/why-you-need-a-mobilelearning-strategy 
Gokhale, A. A. (1995). Collaborative Learning Enhances Critical Thinking. Retrieved on $22^{\text {nd }}$ March, 2013 from http://scholar.lib.vt.edu/ejournals/JTE/v7n1/gokhale.jtev7n1.html

Kumari, M. \& Singh, V. (2009). Mobile Learning: An Emerging Trend. Bombay: Tata Consultancy Services Limited. 1-9.

Mobile Learning (2013). Retrieved on 22 $2^{\text {nd }}$ March, 2013 from http://archive.excellencegateway.org.uk/page.aspx?o=ferl.aclearn.page.id958

Mockus, L., Dawson, H., Edel-Malizia, S., Shaffer, D., Sung An, J., \& Swaggerty, A. (2011). The Impact of Mobile Access on Motivation: Distance Education Students Perceptions. Pennsylvania: Penn State's World Campus. 4-34.

MoLeNET (2012). What is Mobile Learning? Retrieved on $22^{\text {nd }}$ March, 2013 from http://www.molenet.org.uk/

Muyinda, P. B., Lubega, J. T., \& Lynch, K. (2010). Mobile Learning Objects Deployment and Utilization in Developing Countries. International Journal of Computing and ICT Research, 4(1), 37-45.

Ogunlesi, T., \& Busari, S. (2012). Seven Ways Mobile Have Changed Lives in Africa. Retrieved on $27^{\text {th }} \quad$ January, 2013 from http://edition.cnn.com/2012/09/13/world/africa/mobile-phones-changeafrica/index.html

Pieri, M. \& Diamantini, D. (2009). From E-Learning to Mobile Learning: New Opportunities. In Mohamed Ally (Ed.), Mobile Learning: Transforming the Delivery of Education and Training. Edmonton: AU Press. 183-194.

Quesinberry, N. (2011). Why Mobile Learning? Retrieved on 22 ${ }^{\text {nd }}$ March, 2013 from http://learningsolutions.jplcreative.com/blog/index.php/2011/10/31/why-mobilelearning/

RIM (2009). Engaging Higher Education Students with Media-rich Mobile Learning. Chalk Media- Research in Motion whitepaper. Retrieved on $2^{\text {nd }}$ December, 2012 from www.chalk.com

Seehorn Ashley (2013). The Effectiveness of Varying Delivery Methods in Teaching Math. Retrieved on $27^{\text {th }}$ January, 2013 from http://www.ehow.com/m/info8031437_effectiveness_delivery_methods_teaching_math.html

Traxler John (2009). Current State of Mobile Learning. In Mohamed Ally (Ed.), Mobile Learning: Transforming the Delivery of Education and Training. Edmonton: AU Press. 9-21.

Wishart, J. (2009).Use of Mobile Technology for Teacher Training. In Mohamed Ally (Ed.), Mobile Learning: Transforming the Delivery of Education and Training. Edmonton: AU Press. 267-277. 